\title{
Obesity resistance and increased energy expenditure by white adipose tissue browning in $\mathrm{Oga}^{+/-}$mice
}

\author{
Yong Ryoul Yang ${ }^{1}$ - Hyun-Jun Jang ${ }^{1,2} \cdot$ Sun-Sil Choi ${ }^{1}$ Yong Hwa Lee ${ }^{1}$. \\ Gyun Hui Lee ${ }^{1} \cdot$ Young-Kyo Seo ${ }^{1} \cdot J^{\prime}$ ang Hyun Choi ${ }^{1} \cdot$ Dohyun Park ${ }^{2} \cdot$ Ara Koh $^{2}$. \\ Il Shin Kim ${ }^{1} \cdot \mathrm{Ho} \mathrm{Lee}^{3} \cdot$ Sung Ho Ryu ${ }^{2}$ Pann-Ghill Suh ${ }^{1}$
}

Received: 21 April 2015 / Accepted: 4 August 2015 / Published online: 5 September 2015

(C) Springer-Verlag Berlin Heidelberg 2015

\begin{abstract}
Aims/hypothesis $O$-GlcNAcylation plays a role as a metabolic sensor regulating cellular signalling, transcription and metabolism. Transcription factors and signalling pathways related to metabolism are modulated by $\mathrm{N}$-acetyl-glucosamine $(\mathrm{O}-$ GlcNAc) modification. Aberrant regulation of $O$ GlcNAcylation is closely linked to insulin resistance, type 2 diabetes and obesity. Current evidence shows that increased $O$-GlcNAcylation negatively regulates insulin signalling, which is associated with insulin resistance and type 2 diabetes. Here, we aimed to evaluate the effects of $O g a$ (also known as Mgea5) haploinsufficiency, which causes hyper-OGlcNAcylation, on metabolism.

Methods We examined whether $O g a^{+/-}$mice developed insulin resistance. Metabolic variables were determined including body weight, glucose and insulin tolerance, metabolic rate and thermogenesis.

Results $O g a$ deficiency does not affect insulin signalling even at hyper-O-GlcNAc levels. $O g a^{+/-}$mice are lean with reduced
\end{abstract}

Yong Ryoul Yang and Hyun-Jun Jang contributed equally to this study.

Electronic supplementary material The online version of this article (doi:10.1007/s00125-015-3736-z) contains peer-reviewed but unedited supplementary material, which is available to authorised users.

Pann-Ghill Suh

pgsuh@unist.ac.kr

1 School of Life Sciences, Ulsan National Institute of Science and Technology, Building 104, Room 705, UNIST-gil 50, Eonyang-eup, Ulju-gun, Ulsan 689-798, Republic of Korea

2 Department of Life Science, Pohang University of Science and Technology, Pohang, Kyungbuk, Republic of Korea

3 Cancer Experimental Resources Branch, National Cancer Center, Goyang-si, Gyeonggi-do, Republic of Korea fat mass and improved glucose tolerance. Furthermore, $O g a^{+/-}$ mice resist high-fat diet-induced obesity with ameliorated hepatic steatosis and improved glucose metabolism. Oga haploinsufficiency potentiates energy expenditure through the enhancement of brown adipocyte differentiation from the stromal vascular fraction of subcutaneous white adipose tissue (WAT).

Conclusions/interpretation Our observations suggest that $O$-GlcNAcase (OGA) is essential for energy metabolism via regulation of the thermogenic WAT program.

Keywords Adipose tissue browning - Beige adipocyte Obesity $\cdot O$-GlcNAcase $\cdot O$-GlcNAcylation $\cdot$ Thermogenesis

$\begin{array}{ll}\text { Abbreviations } & \\ \text { ACC } & \text { Acetyl-CoA carboxylase } \\ \text { AgRP } & \text { Agouti-related peptide } \\ \text { AMPK } & \text { AMP-activated protein kinase } \\ \text { BAT } & \text { Brown adipose tissue } \\ \text { CLAMS } & \text { Comprehensive Lab Animal Monitoring } \\ & \text { System } \\ \text { GTT } & \text { Glucose tolerance test } \\ \text { HFD } & \text { High-fat diet } \\ \text { ITT } & \text { Insulin tolerance test } \\ \text { MEFs } & \text { Mouse embryo fibroblasts } \\ \text { NButGT } & 1,2 \text {-dideoxy-20-propyl- } \alpha \text {-D-glucopyranoso } \\ & \text {-[2,1-d]- } \delta \text { 20-thiazoline } \\ \text { NC } & \text { Normal chow } \\ \text { NMR } & \text { Nuclear magnetic resonance } \\ \text { OGA } & O \text {-GlcNAcase } \\ O-G l c N A c & N \text {-acetyl-glucosamine } \\ \text { OGT } & O \text {-GlcNAc transferase } \\ \text { PGC1 } \alpha & \text { Peroxisome proliferator-activated receptor } \\ & \gamma \text { coactivator-1- } \alpha\end{array}$




$\begin{array}{ll}\text { PG WAT } & \begin{array}{l}\text { Perigonadal white adipose tissue } \\ O \text {-(2-acetamido-2-deoxy-D- } \\ \text { PUGNAcopyranosylidene) }\end{array} \\ \text { amino- } N \text {-phenylcarbamate } \\ \text { RER } & \begin{array}{l}\text { Respiratory exchange ratio } \\ \text { Inguinal subcutaneous white } \\ \text { adipose tissue }\end{array} \\ \text { SC WAT } & \begin{array}{l}\text { Stromal vascular fraction } \\ \text { Triacylglycerol }\end{array} \\ \text { SVF } & \text { Uncoupling protein 1 } \\ \text { TG } & \text { UDP- } N \text {-acetyl-glucosamine } \\ \text { UCP1 } & \text { Ulsan National Institute of Science } \\ \text { UDP-GlcNAc } & \text { and Technology } \\ \text { UNIST } & \text { White adipose tissue }\end{array}$

\section{Introduction}

$O$-GlcNAcylation is an endpoint in the hexosamine biosynthetic pathway, which produces UDP- $N$-acetyl-glucosamine (UDP-GlcNAc), a donor substrate for $O$-GlcNAc transferase (OGT). Production of UDP-GlcNAc is influenced by the metabolism of carbohydrates, amino acids, fats and nucleotides. $O$-GlcNAcylation modulates diverse cellular processes in response to nutrients. Thus, aberrant $O$-GlcNAcylation has been implicated in many chronic diseases, such as diabetes, obesity, neurodegeneration and cancer [1]. Many studies show that insulin signalling is negatively regulated by the $O$ GlcNAcylation of proteins involved in the insulin signalling pathway $[2,3]$. $O$-GlcNAcylation regulates the activity of transcription factors and cofactors that play key roles in metabolism. Peroxisome proliferator-activated receptor $\gamma$ coactivator-1- $\alpha$ (PGC1 $\alpha)$, CREB-regulated transcription coactivator 2 (CRTC2), forkhead box protein O1 (FOXO1), carbohydrate-responsive element-binding protein (ChREBP) and liver $\mathrm{X}$ receptor $\alpha(\mathrm{LXR} \alpha)$ are $O$-GlcNAcylated, and modification of these proteins regulates gluconeogenic/ lipogenic genes by modulating transcriptional activity in the liver [4-7]. Importantly, the genetic variation in the $O$-GlcNAase ( $\mathrm{Oga}$, also known as Mgea5) gene contributes to increased risk for type 2 diabetes [8]. However, in some populations, $O g a$ is not significantly associated with type 2 diabetes [9].

Mammals have two different types of adipose tissue, white (WAT) and brown adipose tissue (BAT). WAT is the primary site of energy storage, while BAT is a thermogenic tissue. Current evidence shows that environmental conditions induce the development of brown-like adipocytes, the so called beige adipocytes, within certain WAT depots. How the white-tobrown fat transition is regulated is an important issue, as thermogenesis plays a significant role in regulating body weight and protects against diabetes and obesity [10]. In this study, we focused on the role of $O$-GlcNAcase (OGA) in controlling energy expenditure and evaluated the effects of $O g a$ haploinsufficiency in a mouse model.

\section{Methods}

Mice $\mathrm{Oga}^{+/}$mice (C57BL/SV129) were generated as described previously [11] and maintained on the $\mathrm{C} 57 \mathrm{BL} / 6 \mathrm{~J}$ genetic background; $\mathrm{C} 57 \mathrm{BL} / 6 \mathrm{~J}$ breeders were purchased from Jackson Laboratory (Bar Harbor, ME, USA). Mouse strains were bred and housed in the Animal Research Facility at Ulsan National Institute of Science and Technology (UNIST) under specific pathogen-free conditions. Standard chow (A03, Scientific Animal Food \& Engineering, Augy, France) or a high-fat diet (HFD; $60 \%$ of the energy content from fat, D12492; Research Diets, New Brunswick, NJ, USA) and water were provided ad libitum. Experiments were performed after mice were genotyped, without randomisation or blinding. No data were specifically included or excluded. The Institutional Animal Care and Utilization Committee approved all procedures, in accordance with the UNIST Guide for the Care and Use of Laboratory Animals.

Metabolic analysis Body weight was measured weekly, and body composition was assessed using a quantitative nuclear magnetic resonance (NMR) system (EchoMRI100V, Echo Medical Systems, Houston, TX, USA). Oxygen consumption, carbon dioxide production and food intake were measured using Comprehensive Lab Animal Monitoring System (CLAMS; Columbus Instruments, Columbus, OH, USA). For the glucose tolerance test (GTT), mice were given an i.p. injection of D-glucose $(1 \mathrm{~g} / \mathrm{kg}$ body weight $)$ after overnight starvation. For the insulin tolerance test (ITT), mice were fasted for $6 \mathrm{~h}$ and injected with human insulin $(0.75 \mathrm{U} / \mathrm{kg}$ body weight). Serum glucose levels were determined in tail blood samples using a glucometer (Accu-Check Active, Roche, Mannheim, Germany). The serum levels of insulin and leptin were assessed using ELISA kits (Shibayagi, Shibayagi, Gunma, Japan), and triacylglycerol (TG) levels were determined using a colorimetric assay (Cayman Chemical, Ann Arbor, MI, USA). Body temperatures were measured rectally using a digital thermometer (TD-300, Shibaura Electronics, Tokyo, Japan).

\section{Preparation and adipocyte differentiation of stromal vas-} cular fraction cells Stromal vascular fraction (SVF) cells were isolated from the inguinal adipose tissue of 5-6-weekold mice. Inguinal adipose tissue was excised and minced in $10 \mathrm{ml}$ digestion buffer $(100 \mathrm{mmol} / \mathrm{l}$ HEPES pH7.4, 120 $\mathrm{mmol} / \mathrm{l} \mathrm{NaCl}, 50 \mathrm{mmol} / \mathrm{l} \mathrm{KCl}, 5 \mathrm{mmol} / \mathrm{l}$ glucose, $1 \mathrm{mmol} / \mathrm{l}$ $\mathrm{CaCl}_{2}, 1.5 \%$ BSA-fatty acid free and $1 \mathrm{mg} / \mathrm{ml}$ collagenase II), and the tissue was incubated at $37^{\circ} \mathrm{C}$ with shaking for $2 \mathrm{~h}$. Larger particles were removed using a $100 \mu \mathrm{m}$ cell 
strainer, and $30 \mathrm{ml}$ SVF medium (DMEM/F12 [1:1], 10\% FBS, Glutamax and Pen/Strep) was added to filtrates. The filtrates were centrifuged at $600 \mathrm{~g}$ for $5 \mathrm{~min}$ to remove floating adipocytes. The pelleted SVF cells were resuspended in $10 \mathrm{ml}$ SVF medium and passed through a $40-\mu \mathrm{m}$ cell strainer. Adipocyte differentiation of confluent SVF cells was induced after 2 days using $5 \mu \mathrm{g} / \mathrm{ml}$ insulin, $5 \mu \mathrm{mol} / 13$-isobutyl-1methylxanthine, $5 \mu \mathrm{mol} / 1$ dexamethasone, $1 \mathrm{nmol} / 1$ 3,3',5triiodo-L-thyronine, $125 \mu \mathrm{mol} / \mathrm{l}$ indometacin and $1 \mu \mathrm{mol} / 1$ rosiglitazone. The cells were kept in differentiation medium containing $5 \mu \mathrm{g} / \mathrm{ml}$ insulin, $1 \mathrm{nmol} / 1$ 3,3',5-triiodo-L-thyronine and $1 \mu \mathrm{mol} / 1$ rosiglitazone for an additional 6 days. The medium was changed every 2 days.

\section{Adipocyte differentiation of mouse embryo fibroblasts} Mouse embryo fibroblasts (MEFs) were harvested from 12.5-14.5 days post coitum embryos as described previously [11]. MEFs were propagated to confluence. Two days later, adipocyte differentiation was induced by treatment with $5 \mu \mathrm{g} / \mathrm{ml}$ insulin, $5 \mu \mathrm{mol} / 1$ 3-isobutyl-1-methylxanthine, $5 \mu \mathrm{mol} / \mathrm{l}$ dexamethasone and $125 \mu \mathrm{mol} / \mathrm{l}$ indometacin. This medium was changed every other day. After 4 days, the induction medium containing $5 \mu \mathrm{g} / \mathrm{ml}$ insulin was used to maintain differentiation.

Western blotting Tissue and cell lysates were prepared using standard procedures. Protein samples $(20-30 \mu \mathrm{g})$ were separated on an SDS-polyacrylamide gel and visualised. The antibodies used are listed in Electronic Supplementary Material (ESM) Methods.

Real-time PCR analysis Real-time PCR analysis and the primers used are listed in ESM Methods and in ESM Table 1.

Statistical analysis Data are presented as the mean \pm SEM or $\mathrm{SD}$ as indicated in the figure legends. Comparisons between two groups were performed using unpaired two-tailed Student's $t$ tests. A p value $<0.05$ was considered statistically significant.

\section{Results}

Increased $\boldsymbol{O}$-GlcNAcylation has no effect on insulin signalling Many studies show that increased $O$-GlcNAcylation by OGT overexpression or $O$-(2-acetamido-2-deoxy-Dglucopyranosylidene) amino- $N$-phenylcarbamate (PUGNAc) treatment perturbs activation of insulin signalling. To investigate whether elevated $O$-GlcNAcylation by $O g a$ deletion affects insulin signalling, we treated $O g a^{+/+}$and $O g a^{-/-}$MEFs with insulin. Phosphorylation of IRS-1 at Tyr608 and of Akt at Thr308/Ser473 triggered by insulin was not perturbed in $O g a^{-/-}$ MEFs with high levels of $O$-GlcNAcylation (Fig. 1a). To further confirm the effect of increased $O$-GlcNAcylation on insulin signalling, we used $O g a^{+/-}$mice. We previously reported neonatal lethality in $O g a^{-/-}$mice [11]. However, $O g a^{+-}$mice are viable and fertile, and western blot analysis showed increased $O$-GlcNAcylation in metabolic tissues, liver, muscle, and WAT in $O g a^{+/}$mice compared with $O g a^{+/+}$mice (Fig. 1b). $O g a^{+/+}$and $O g a^{+/-}$mice were starved and injected i.p. with insulin. Insulin-stimulated Akt phosphorylation in the liver, muscle and WAT of $O g a^{+/-}$mice was not suppressed by increased $O$-GlcNAcylation signalling (Fig. 1c).

$\mathrm{Oga}^{+/-}$mice have a lean phenotype and reduced fat mass $\mathrm{Oga}^{+/-}$mice were apparently smaller than $O g a^{+/+}$mice throughout development after birth. $O g a^{+/}$mice fed normal chow (NC) showed significant decreases in body weight resulting in a lean phenotype (Fig. 2a). Importantly, the percentage of fat mass in $\mathrm{Oga}^{+/+}$mice was significantly reduced, whereas lean mass was comparable between $\mathrm{Oga}^{+/+}$and $\mathrm{Oga}^{+/}$mice (Fig. 2b). Weights of BAT in $\mathrm{Oga}^{+/-}$mice were unchanged compared with $O G A^{+/+}$mice. Lipid content of perigonadal (PG) WAT appeared to be slightly lower in $\mathrm{Oga}^{+/-}$mice than $\mathrm{Oga}^{+/+}$mice, but the difference was not significant. In contrast, lipid content in inguinal subcutaneous (SC) WAT was reduced in $\mathrm{Oga}^{+/}$mice (Fig. 2c). There was no significant difference in cell size in the BAT and PG WAT between $\mathrm{Oga}^{+/+}$and $O g a^{+/-}$mice. Notably, the SC WAT of $\mathrm{Oga}^{+/}$mice showed islets of multilocular brown fat-like adipocytes (Fig. 2d).

Previous data show that increased $O$-GlcNAcylation due to an OGA inhibitor, 1,2-dideoxy-20-propyl- $\alpha$-Dglucopyranoso-[2,1-d]- $\delta$ 20-thiazoline (NButGT), suppresses adipocyte differentiation through modulation of PPAR $\gamma$ transcriptional activity [12]. To assess whether the reduced WAT weight in $\mathrm{Oga}^{+/}$mice results from a decrease in adipocyte number due to impaired differentiation, we compared the adipocyte differentiation capacity of MEFs isolated from $O g a^{+/+}$ and $O g a^{-/}$embryos. After undergoing differentiation into adipocytes, we observed no differences in accumulation of intracellular lipids as determined using Oil Red O staining (Fig. 2e,f). This result suggests that the reduced WAT weight of $O g a^{+-}$mice does not reflect impaired adipogenesis of precursor cells.

Improved glucose tolerance in $\boldsymbol{O} \boldsymbol{g} \boldsymbol{a}^{+/-}$mice We next investigated whether $O g a$ heterozygosity affects glucose homeostasis. No changes in blood glucose level, serum insulin level, or serum leptin were observed between $O g a^{+/+}$and $O g a A^{+/-}$mice under fasting conditions (Fig. 3a-c). The GTT showed that glucose levels in $\mathrm{Oga}^{+/-}$mice remained significantly lower than those of control $\mathrm{Oga}^{+/+}$mice after glucose injection, indicating that glucose tolerance was better in $\mathrm{Oga}^{+/}$mice than $O \mathrm{ga}^{+/+}$mice (Fig. 3d). In contrast, no difference was observed 
Fig. 1 Elevation of $O$ -

GlcNAcylation has no effect on insulin signalling. (a) $\mathrm{Oga}^{+/+}$and $\mathrm{Oga}^{-/-}$MEFs were serum starved for $12 \mathrm{~h}$ and stimulated with $100 \mathrm{nmol} / \mathrm{l}$ insulin for the indicated times (in $\mathrm{min}$ ). (b) Western blot analysis of $O$ GlcNAcylation and OGA levels in WAT, liver and muscle lysates from $O g a^{+/+}$and $O g a^{+/-}$mice. (c) Western blot analysis of phospho (Ser 473), phospho (Thr 308) and total Akt protein levels in the liver, muscle and WAT of $O g a^{+/+}$ and $O g a^{+/-}$mice with or without insulin administration; numbers indicate individual mice in each group a

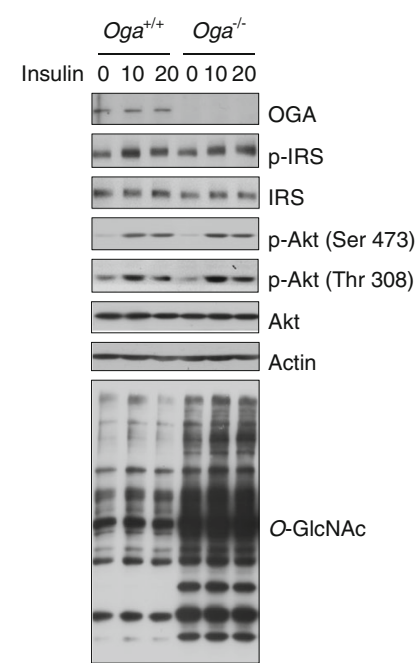

b

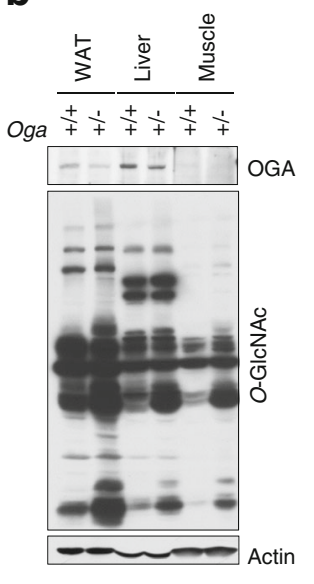

C
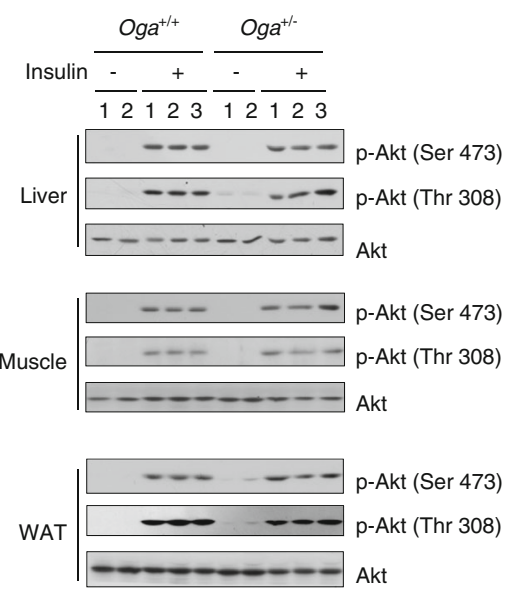

in insulin sensitivity between $O g a^{+/+}$and $O g a^{+/-}$mice as measured by the ITT, indicating that hepatic insulin sensitivity was unaffected in $\mathrm{Oga}^{+/-}$mice (Fig. 3e). To determine whether Oga heterozygosity affects gluconeogenesis and lipid metabolism, we measured the mRNA expression of key gluconeogenic (Pepck [also known as Pckl], G6p [also known as H6pd] and Pgcl $\alpha$ [also known as Ppargcla]) and fatty acid metabolism-related genes (Fasn, Mcad [also known as Acadm], Scd1 and Srebp-1c [also known as Srebf1]). Notably, mRNA levels of hepatic Pepck and G6p were
Fig. $2 \mathrm{Oga}^{+/-}$mice are lean and have reduced lipid content. (a) Weight gain of male littermates fed a chow diet $(n=8-12)$; white circles, $\mathrm{Oga}^{+/+}$; black circles, $\mathrm{Oga}^{+/-}$. (b) Body composition of $\mathrm{Oga}^{+/+}$(white bars) and $O g a^{+/-}$ (grey bars) mice at 8 weeks of age; total weight, fat mass, lean mass and total body water on a chow diet were determined by quantitative NMR $(n=5)$. (c) PG, $\mathrm{SC}, \mathrm{BAT}$ and perirenal fat depot weights in 10-week-old $\mathrm{Oga}^{+/+}$ and $O \mathrm{ga}^{+/-}$male mice fed a chow $\operatorname{diet}(n=5)$. (d) Histological analysis of adipose tissues from 10-week-old $O g a^{+/+}$and $O g a^{+/-}$ male mice fed a chow diet. Scale bar, $100 \mu \mathrm{m}$. (e) Oil Red O staining of $\mathrm{Oga}^{+/+}$and $\mathrm{Oga}^{-/-}$ MEFs before induction of adipocyte differentiation (day 0) and after complete differentiation (day 7). (f) Relative absorbance of Oil Red $\mathrm{O}$ after adipocyte differentiation. Error bars represent the SD. $\uparrow p<0.05$, $\dagger \uparrow p<0.005, \uparrow \dagger \uparrow p<0.0005$ (Student's $t$ test) a

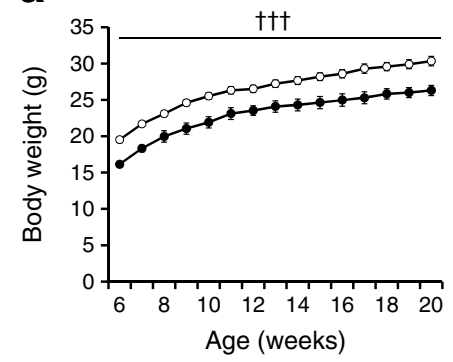

b

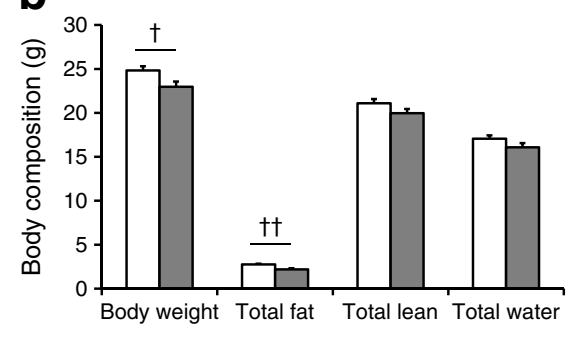

C

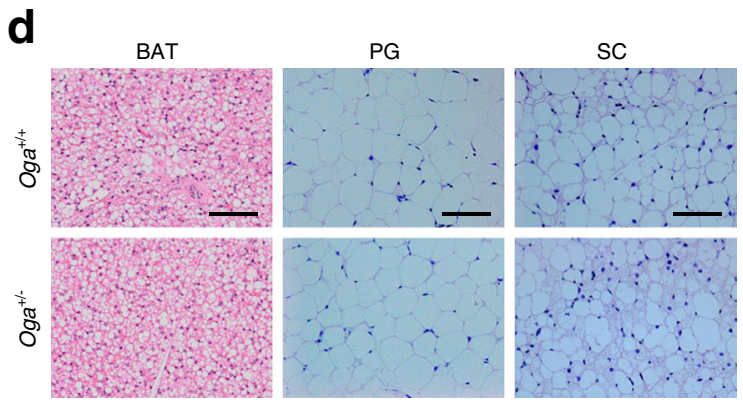

e

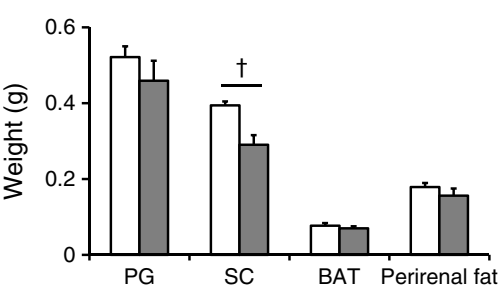<smiles>[13CH3]</smiles>
Induction
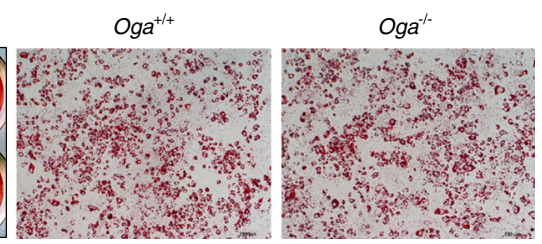

Oil Red O f

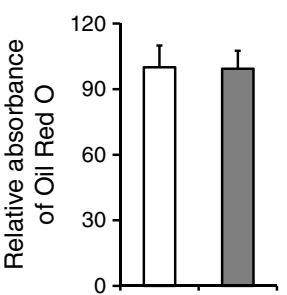



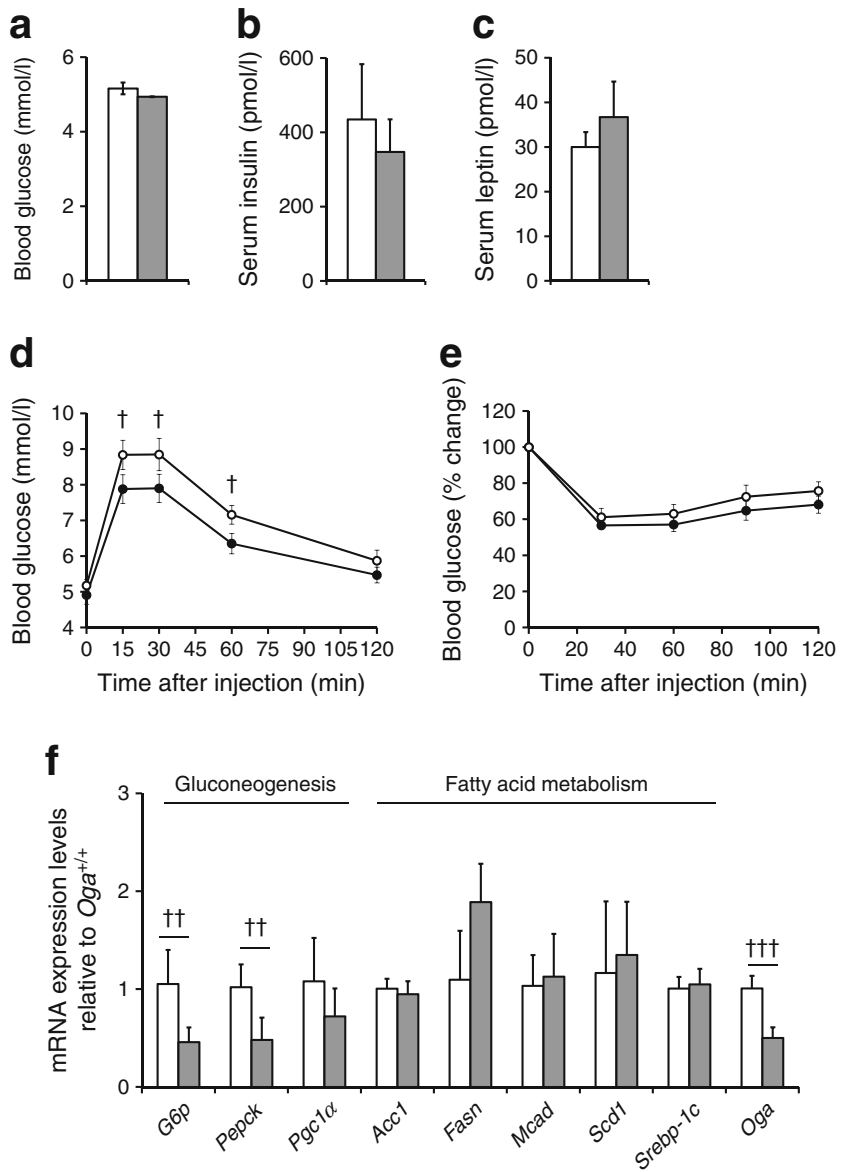

Fig. 3 Improved glucose tolerance in $\mathrm{Oga}^{+/-}$mice. (a) Blood glucose, (b) serum insulin and (c) serum leptin levels were measured after a $16 \mathrm{~h}$ fast in 10-week-old male $\mathrm{Oga}^{+/+}$(white bars) and $O g a^{+/-}$mice (grey bars) fed a chow diet $(n=8)$. (d) GTT and (e) ITT in 10-week-old male $O g a^{+/+}$ (white circles) and $O g a^{+-}$mice (black circles) fed a chow diet $(n=8)$. Error bars represent the SEM. $\uparrow p<0.05$ (Student's $t$ test). (f) Expression of gluconeogenesis- and fatty acid metabolism-related genes in the liver as determined by real-time quantitative PCR. Relative mRNA expression was normalised to Gapdh. White bars, $O g a^{+/+}$; grey bars, $O g a^{+/-}$. Error bars represent the SD. $\dagger \uparrow p<0.005, \uparrow \dagger p<0.0005$ (Student's $t$ test)

reduced in $\mathrm{Oga}^{+/-}$mice compared with $O g a^{+/+}$mice. In contrast, $P g c l \alpha$ levels were not altered in $O g a^{+/-}$mice. These results indicate that improved glucose tolerance is reflected by reduced expression of gluconeogenesis genes in $\mathrm{Oga}^{+/-}$ mice. With regard to fatty acid metabolism, no significant difference was observed between $O g a^{+/+}$and $O g a^{+/-}$mice (Fig. 3f).

Increased energy expenditure in $\boldsymbol{O g a}{ }^{+/-}$mice Body weight is determined by the balance between food intake and energy expenditure. Therefore, we speculated that energy expenditure might be elevated in $O g a^{+/-}$mice compared with $O g a^{+/+}$ mice. Nutrients were burned more efficiently in $\mathrm{Oga}^{+/-}$ mice than in $\mathrm{Oga}^{+/+}$mice. Increased body oxygen consumption (Fig. 4a,b) and $\mathrm{CO}_{2}$ production (Fig. 4c,d) were observed in $\mathrm{Oga}^{+/-}$mice during dark and light periods compared with $\mathrm{Oga}^{+/+}$mice. The rate of food intake per day was not significantly different between $\mathrm{Oga}^{+/+}$and $\mathrm{Oga}^{+/-}$mice (Fig. 4e). The increased metabolic rate was not due to increased physical activity (Fig. 4f,g). Heat production was not significantly altered in $\mathrm{Sg}^{+/-}$mice compared with wild-type mice (Fig. 4h).

Several studies suggested that elevation of $O$-GlcNAcylation enhances fatty acid oxidation through activation of AMPactivated protein kinase (AMPK), a key player regulating energy balance at both the cellular and whole-body levels $[13,14]$. We examined the phosphorylation of AMPK and acetyl-CoA carboxylase (ACC) in muscle, liver and WAT isolated from $\mathrm{Oga}^{+/+}$ and $O g a^{+/-}$mice. There were no significant differences in phosphorylation of AMPK or ACC (ESM Fig. 1).

$\mathrm{Oga}^{+/-}$mice were resistant to HFD-induced obesity and showed improved glucose tolerance and insulin sensitivity To examine the effects of $O g a$ heterozygosity on HFDinduced obesity and insulin resistance, we challenged $O g a^{+/}$ mice with a HFD for 12 weeks. On the HFD, $O g a^{+/+}$mice became obese, whereas $\mathrm{Oga}^{+/-}$mice were quite resistant to obesity. Body weight accumulation was lower in $\mathrm{Oga}^{+/}$mice than in $\mathrm{Oga}^{+/+}$mice (Fig. 5a). The fat mass of $\mathrm{Oga}{ }^{+/-}$mice fed a HFD was significantly smaller than that of $O g a^{+/+}$mice (Fig. 5b). Notably, the weights of SC WAT, PG WAT and BAT of $\mathrm{Oga}^{+/-}$mice were lower than those of $\mathrm{Oga}^{+/+}$mice. The weight of PG WAT in $\mathrm{Oga}^{+/-}$mice was slightly reduced compared with $O g a^{+/+}$mice, but the difference was not significant (Fig. 5c). Histological analysis revealed no significant differences in white adipocyte size between $O g a^{+/+}$mice and $\mathrm{Oga}^{+/}$mice. In contrast, $\mathrm{Oga}^{+/}$mice showed reduced lipid accumulation in BAT and liver compared with $O g a^{+/+}$mice. Moreover, haematoxylin and eosin staining and Oil Red O staining revealed that liver steatosis was much lower in $\mathrm{Oga}^{+/-}$mice than $\mathrm{Oga}^{+/+}$mice (Fig. 5d). Consistent with the lean phenotype, blood glucose, serum TG, insulin and leptin levels in $\mathrm{Oga}^{+/-}$mice were significantly reduced relative to $\mathrm{Oga}^{+/+}$mice during the HFD feeding period (Fig. 5e, ESM Fig. 2a-c). We next assessed glucose homeostasis and insulin sensitivity. $O g a^{+/-}$mice showed improved glucose tolerance and increased insulin sensitivity compared with $\mathrm{Oga}^{+/+}$mice on a HFD (Fig. $5 \mathrm{f}$ and $5 \mathrm{~g}$ ). To determine whether $\mathrm{Oga}^{+/-}$ mice fed a HFD also exhibited increased energy expenditure, we monitored the metabolic activities of these mice using CLAMS. $\mathrm{Oga}^{+/-}$mice displayed increased oxygen consumption (Fig. 5h,i) and $\mathrm{CO}_{2}$ production (ESM Fig. 3a,b) on the HFD. Food intake did not significantly differ between $O g a^{+/+}$mice and $\mathrm{Oga}^{+/-}$mice (ESM Fig. 3c). Despite the increased energy expenditure, locomotor activity was decreased in $\mathrm{Oga}^{+/}$mice (ESM Fig. 3d,e). Heat production was slightly, but not significantly, increased in $\mathrm{Oga}^{+/-}$mice (ESM Fig. 3f). 
Fig. 4 Increased energy expenditure in $O g a^{+-}$mice. $(\mathbf{a}, \mathbf{b})$ $\mathrm{O}_{2}$ consumption rate $\left(\dot{V} \mathrm{O}_{2}\right),(\mathbf{c}, \mathbf{d})$ $\mathrm{CO}_{2}$ consumption rate $\left(\dot{V} \mathrm{O}_{2}\right),(\mathbf{e})$ food intake and (f, g) locomotor activity measured using CLAMS in 10-week-old mice fed a chow diet. One $24 \mathrm{~h}$ period is shown $(n=$ 4). (h) Rectal temperatures of $\mathrm{Oga}^{+/+}$and $\mathrm{Oga}^{+/-}$mice fed a chow diet $(n=7)$. White circles and white bars, $O g a^{+/+}$; black circles and grey bars, $\mathrm{Oga}^{+/-}$. Grey areas indicate dark periods (18:00 hours to 06:00 hours). Error bars represent the SEM. $\dagger \dagger p<0.005$ (Student's $t$ test)


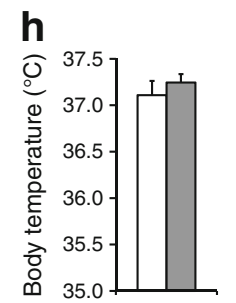

Enhanced differentiation of subcutaneous WAT-derived SVF cells into brown-like adipocytes in $\boldsymbol{O g a}^{+-}$mice To determine the relative levels of $O$-GlcNAc cycling enzymes and $O$-GlcNAcylation levels in BAT and WATs, we isolated BAT, PG and SC WAT from wild-type mice. OGT and OGA expression levels were higher in SC WAT than in BAT and PG WAT, and the levels of $O$-GlcNAcylation were elevated in PG WAT and SC WAT compared with BAT (Fig. 6a). The weight (Fig. 2c) and histological morphology of BAT (Fig. 2d) did not differ between $O g a^{+/+}$mice and $O g a^{+/-}$mice. Notably, the weight of SC WAT in $\mathrm{Oga}^{+/-}$mice was lower than that in $\mathrm{Oga}^{+/+}$mice (Fig. 2c). In addition, SC WAT in $\mathrm{Oga}^{+/-}$mice contained clusters of brown-like adipocytes (multilocular adipocytes), also known as beige fat cells (Fig. 2d). The thermogenic gene program of beige fat cells elevates energy expenditure leading to body weight loss [10]. The thermogenic genes were significantly increased in SC WAT in $O g a^{+/-}$mice (Fig. 6b). In PG WAT, Pgcla and Elovl3 expression levels were elevated in $\mathrm{Oga}^{+/-}$mice compared with $\mathrm{Oga}^{+/+}$mice (Fig. 6c). However, there was no significant difference in BAT between $O g a^{+/+}$and $O g a^{+/-}$mice (Fig. 6d).

To investigate the function of OGA in the thermogenic adaptation of SC WAT, we exposed wild-type mice to cold temperatures. The OGA protein was decreased in the SC WAT of cold-exposed mice concomitant with increased PGC1 $\alpha$ and uncoupling protein 1 (UCP1) proteins, while OGT protein was not significantly altered, suggesting an important role for OGA in thermogenic adaptation (Fig. 6e). To assess the effect of $O g a$ haploinsufficiency in thermogenesis, we challenged $\mathrm{Oga}^{+/+}$and $O g \mathrm{~g}^{+/-}$mice using cold temperatures. Although core body temperature did not differ between $\mathrm{Oga}^{+/+}$and $O g \mathrm{ga}^{+/-}$mice at $23^{\circ} \mathrm{C}, O g \mathrm{O}^{+/-}$mice had higher body temperatures than those of $\mathrm{Oga}^{+++}$mice at $4^{\circ} \mathrm{C}$ (Fig. 6f). Consistent with these results, PGC1 $\alpha$ and UCP1 expression levels in SC WAT of $O g a^{+-}$mice are markedly increased compared with $\mathrm{Oga}^{+/+}$mice (Fig. 6g).

The SVF contains fat progenitor cells that have the potential to differentiate into brown-like adipocytes $[10,15]$. This prompted us to examine whether the differentiation of SVF from SC WAT into brown-like adipocytes is enhanced in $\mathrm{Oga}^{+/-}$mice. We isolated SVF from SC WAT of $\mathrm{Oga}^{+/+}$and $O g a^{+/-}$mice, followed by induction of adipocyte differentiation. Both groups of SVF efficiently differentiated into adipocytes, as indicated by Oil Red O staining (Fig. 6h). Notably, brown-like adipocyte genes-Pgcla, Ucpl, Cidea and $C o x 5 b$-were significantly increased in the differentiated $O g a^{+/-}$SVF compared with $O g a^{+/+}$SVF (Fig. 6i,j). To evaluate the effects of increased $O$-GlcNAcylation on the differentiation of SVF, adipose SVF cells were treated with Thiamet-G, a potent and selective OGA inhibitor [16]. However, ThiametG treatment did not affect brown adipocyte differentiation of SVF cells (ESM Fig. 4a). Notably, increased $O$-GlcNAcylation by Thiamet-G treatment resulted in elevated OGA expression (ESM Fig. 4b), as 
a

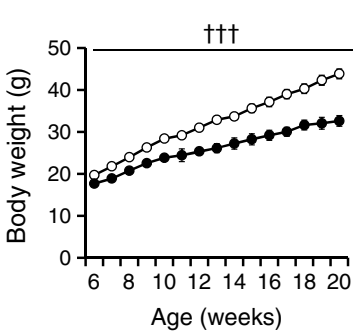

C

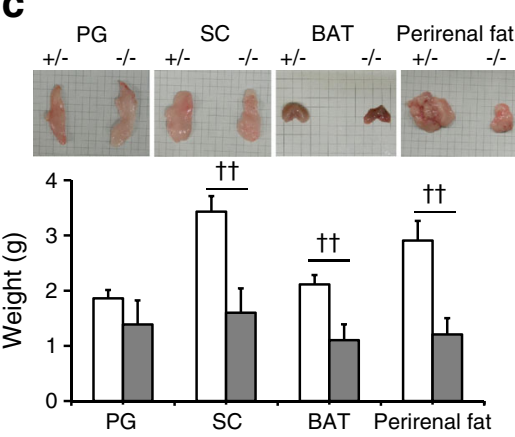

b

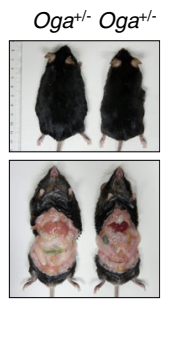

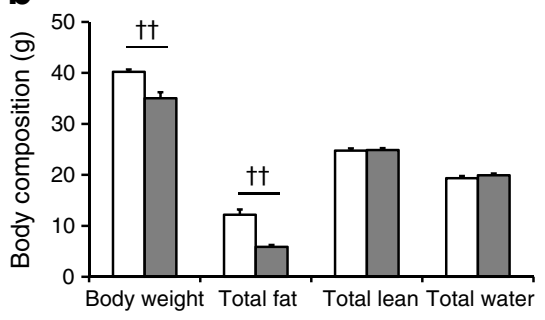

d
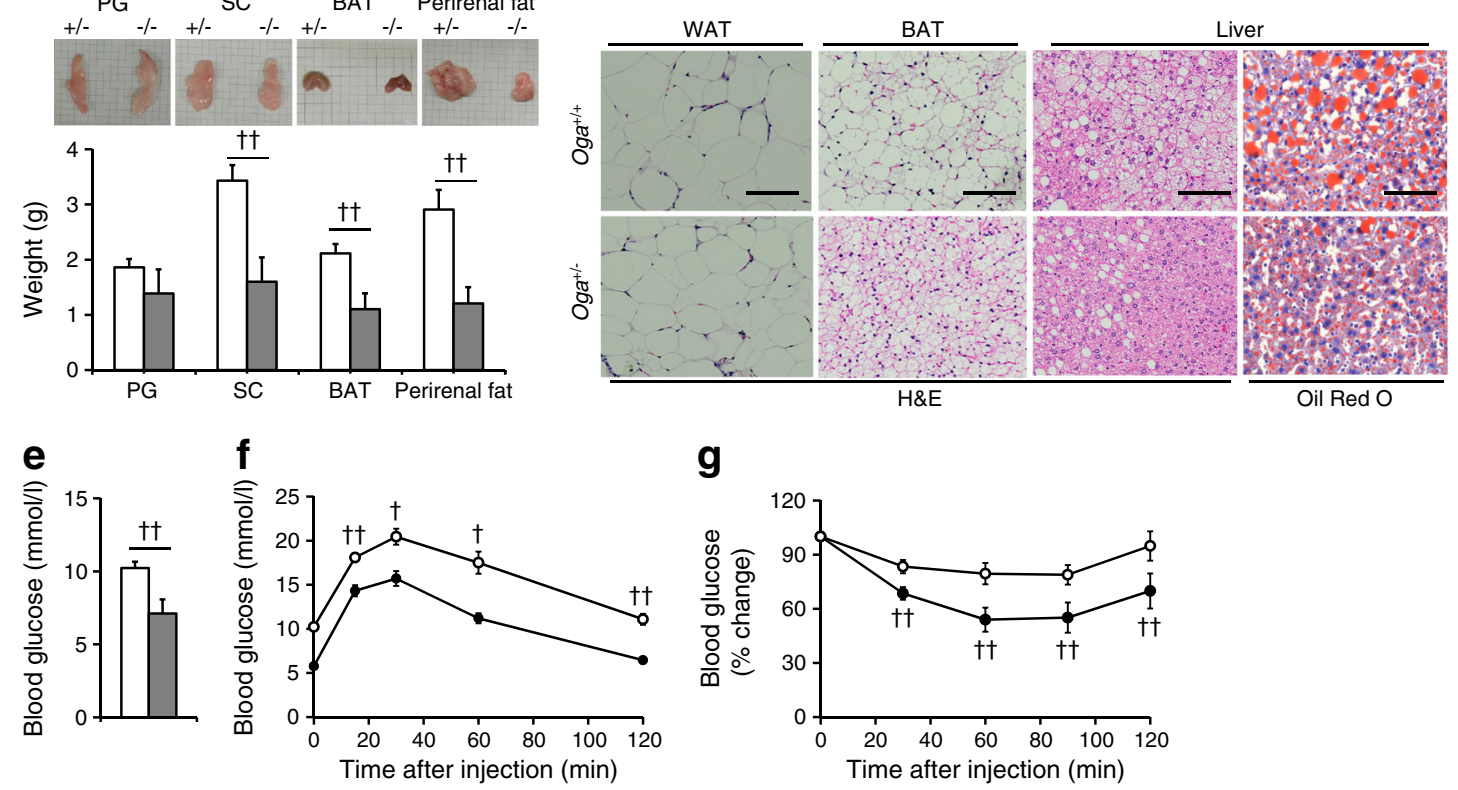

g
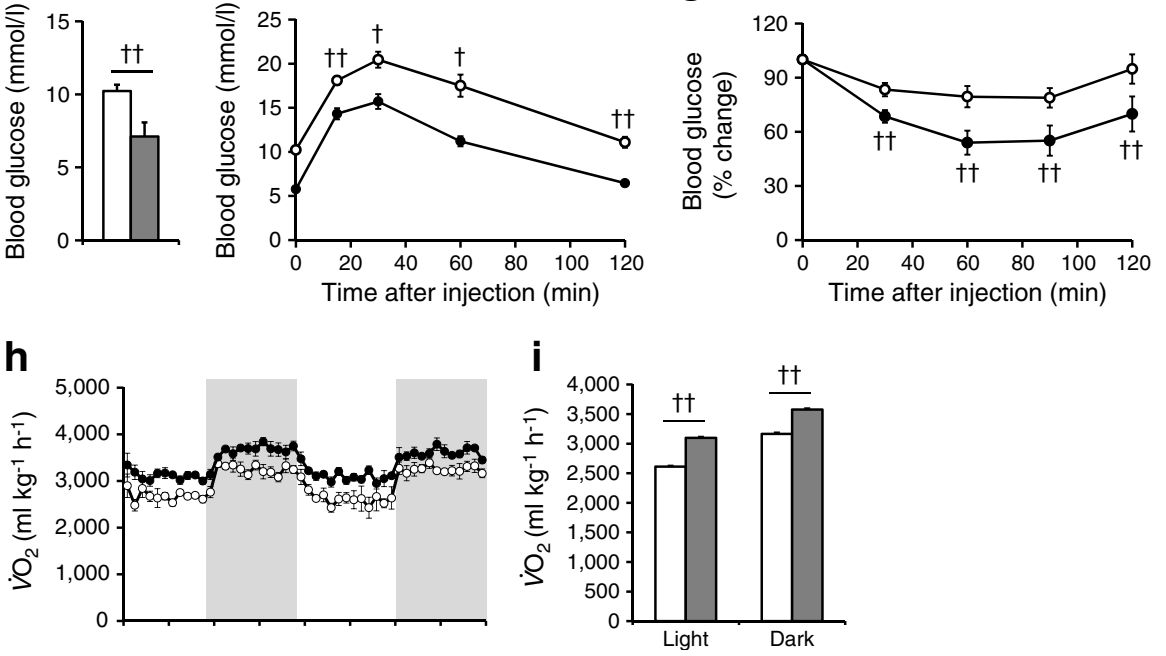

Fig. $5 \mathrm{Oga}^{+/-}$mice display resistance to HFD-induced obesity. (a) Weight gain in male littermates fed an HFD for 12 weeks $(n=10-12)$. Gross appearance of 18 -week-old $\mathrm{Oga}^{+/+}$and $O g a^{+/-}$mice after HFD feeding. (b) Body composition of $O g a^{+/+}$and $O g a^{+/-}$mice $(n=5)$. (c) PG, SC, BAT and perirenal fat depot weights in 18 -week-old $O g a^{+++}$ and $\mathrm{Oga}^{+-}$male mice $(n=5)$. (d) Histological analysis of adipose tissue and liver, and Oil Red O staining of livers. Scale bar, $100 \mu \mathrm{m}$. H\&E,

previously observed [17]. These results indicate that the control of OGA protein expression is required for differentiation of SVF.

To further observe synergistic effects, we evaluated differentiated SVF in the presence of forskolin, an adenylyl cyclase activator. Activation of cAMP triggers protein kinase A (PKA) activation, which induces the expression of thermogenic genes [18]. mRNA levels of Pgcla and Cox5b were markedly elevated in the differentiated $O g a^{+/-}$SVF compared haematoxylin and eosin staining. (e) Blood glucose levels were measured $(n=8)$. (f) GTT and (g) ITT $(n=8)$. $(\mathbf{h}, \mathbf{i}) \mathrm{O}_{2}$ consumption rate $\left(\dot{V} \mathrm{O}_{2}\right)$ measured using CLAMS; one $24 \mathrm{~h}$ period is shown $(n=4)$. Grey areas indicate dark periods (18:00 hours to 06:00 hours). White circles and white bars, $\mathrm{Oga}^{+/+}$; black circles and grey bars, $O g a^{+/-}$. Error bars represent the SEM. $\dagger p<0.05, \dagger \dagger p<0.005, \dagger \dagger \dagger p<0.0005$ (Student's $t$ test)

with $O g a^{+/+}$SVF (Fig. 6k). Overall, these results suggest that Oga heterozygosity enhances brown adipocyte differentiation of SVF from SC WAT.

\section{Discussion}

In this study, we demonstrated the important role of OGA in controlling energy expenditure in vivo using $O g a^{+/-}$mice. 


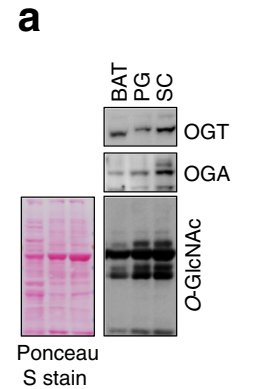

e

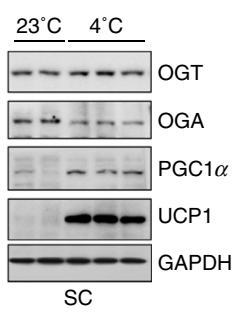

b

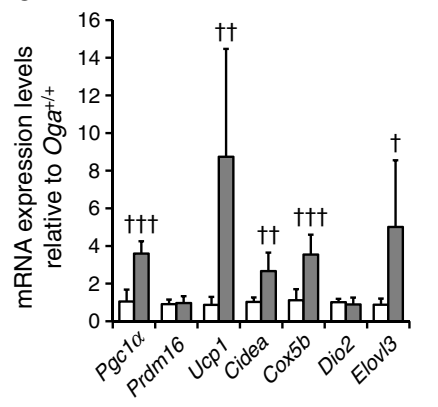

f

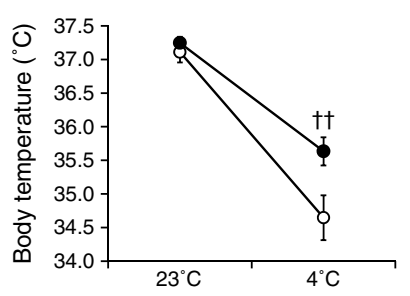

C

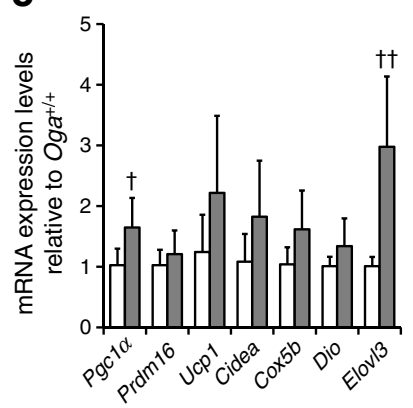

d

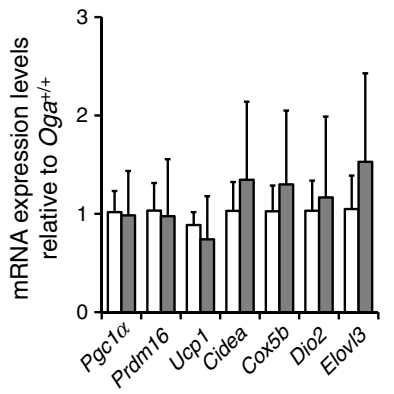

g

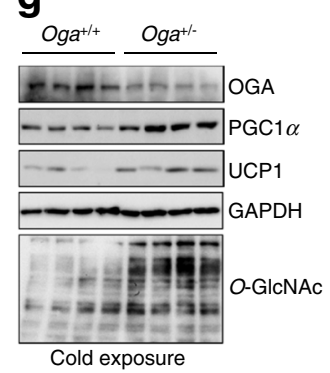

h
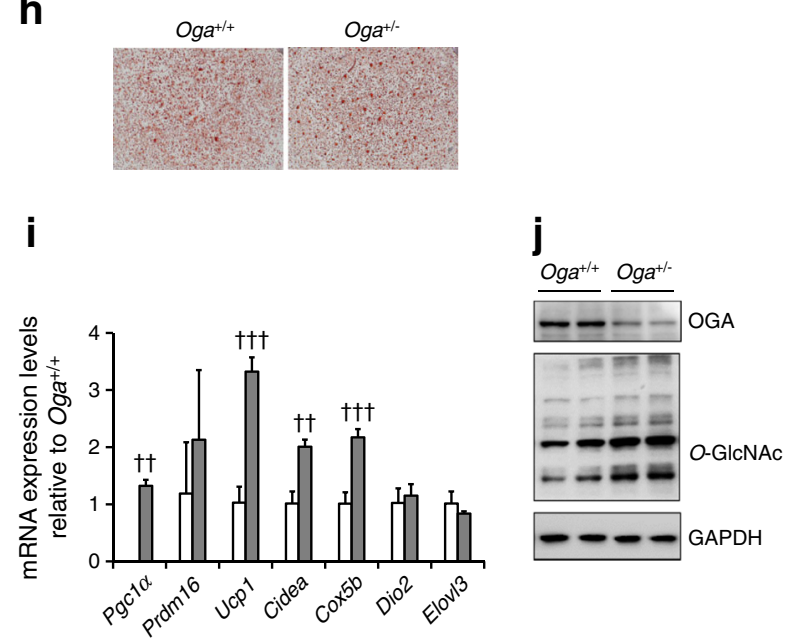

k

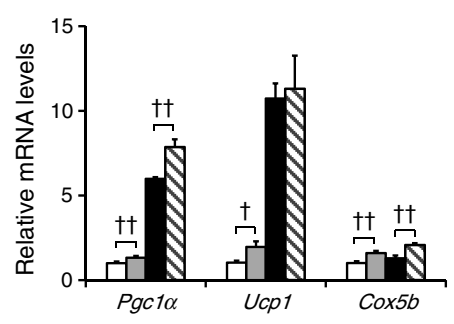

Fig. 6 Enhanced differentiation of SVF from subcutaneous WAT. (a) Expression levels of $O$-GlcNAc enzymes and $O$-GlcNAcylation in BAT, PG and SC WAT. (b-d) mRNA expression of classic brown fat markers in SC WAT (b), PG WAT (c) and BAT (d), respectively, in $\mathrm{Oga}^{+/+}$(white bars) vs $O g a^{+/-}$mice (grey bars) $(n=5)$. Relative mRNA expression was normalised to Gapdh. Error bars represent the SD. (e) Western blot analysis of $O$-GlcNAc enzymes in SC WAT of wild-type mice maintained at either $23^{\circ} \mathrm{C}$ or $4^{\circ} \mathrm{C}$. (f) Body temperature of $O g a^{+/+}$vs $\mathrm{Oga}^{+-}$mice. Results represent average body temperature over a $48 \mathrm{~h}$ period at $23^{\circ} \mathrm{C}$ or $4^{\circ} \mathrm{C}(n=10)$. White circles, $O g a^{+/+}$; black circles, $\mathrm{Oga}^{+-}$. (g) Western blot analysis of PGC1 $\alpha$ and UCP1 in SC WAT of

Importantly, we observed that increased $O$-GlcNAcylation does not affect insulin signalling. $O g a^{+/-}$mice are lean, with reduced body fat mass and resistance to diet-induced obesity, which results from enhanced brown adipocyte differentiation in SC WAT. Our findings thus provide novel insight in to the role of OGA in thermogenesis.

$\mathrm{Oga}^{+/+}$vs $\mathrm{Oga}^{+/-}$mice after cold exposure $\left(7\right.$ days, $\left.4^{\circ} \mathrm{C}\right)$. Error bars represent the SEM. (h) Oil Red O staining and (i) relative mRNA levels of BAT makers in the differentiated SVF cells from $\mathrm{Oga}^{+/+}$and $O g a^{+/-}$ mice after differentiation for 8 days with classical white adipocyte induction. Relative mRNA expression was normalised to Gapdh. (j) Western blot analysis of OGA and $O$-GlcNAc levels in differentiated SVF cells from $\mathrm{Oga}^{+/+}$and $O g a^{+/-}$mice. (k) Relative mRNA levels of Pgcl $\alpha, U c p 1$ and Cox $5 b$ in fully differentiated SVF $(n=3)$. Relative mRNA expression was normalised to Gapdh. Error bars represent the SD. White bars, $\mathrm{Oga}^{+/+}$; grey bars, $\mathrm{Oga}^{+/-}$; black bars, $\mathrm{Oga}^{+/+}+$forskolin; hatched bars, $O g a^{+/-}+$forskolin. $\uparrow p<0.05, \dagger \dagger p<0.005, \dagger \dagger \uparrow p<0.0005$ (Student's $t$ test)

We found that increased $O$-GlcNAcylation through genetic deletion of $O g a$ does not affect insulin signalling or lead to insulin resistance. However, accumulating evidence suggests that increased $O$-GlcNAcylation leads to insulin resistance by negatively regulating insulin signalling. Elevation of $O$ GlcNAcylation by PUGNAc (an OGA inhibitor) suppresses 
insulin signalling in cultured cells [19]. In addition to results from in vitro studies, researchers found that increased levels of $O$-GlcNAcylation lead to insulin resistance in vivo $[2,20]$. PUGNAc is a potent inhibitor of OGA; however, it has some off-target effects $[21,22]$. Several papers show that other OGA inhibitors, such as NButGT, do not affect insulin signalling [23, 24]. Moreover, NButGT also does not disturb insulin signalling in vivo [25]. These complex results were also observed in nuclear factor $\mathrm{kB}(\mathrm{NF}-\mathrm{kB})[26,27]$ and $\mathrm{Wnt}$ signalling $[17,28]$. The different effects of the changes in $O$-GlcNAc levels might result from the different methods used to elevate $O$-GlcNAcylation.

We found that $O g a$ haploinsufficiency enhances brown adipocyte differentiation in WAT SVF. Many factors that regulate brown adipocyte differentiation, such as PGC1 $\alpha$ [29], PPAR $\gamma[30]$ and $\beta$-catenin [31], have been identified. Importantly, these factors were $O$-GlcNAcylated $[12,32$, 33]. Thus, we speculate that the increased brown adipocyte differentiation in $O g a^{+/}$mice might be due to the altered activity of key factors that are $O$-GlcNAcylated or form complexes with $O$-GlcNAc cycling enzymes.

Recently, the phenotype of different $O g a^{+/-}$mice lines was analysed [34]. Keembiyehetty et al generated $O g a$ knockout mice by crossing floxed $O g a$ mice with murine mammary tumour virus (MMTV)-Cre mice, which express Cre recombinase in the murine zygote. We used $O g a^{+/+}$and $\mathrm{Oga}^{+/-}$male mice in all experiments. $O g a^{+/-}$female mice also had significantly lower body weight than $O g a^{+/+}$female mice (ESM Fig. 5). In contrast, Keembiyehetty et al observed different results, showing that the body weight of $O g a^{+/+}$and $\mathrm{Oga}^{+/-}$male mice were comparable, whereas $\mathrm{Oga}^{+/-}$female mice showed increased weight gain compared with $\mathrm{Oga}^{+/+}$ mice on NC and high-fat chow. As Keembiyehetty et al reported increased respiratory exchange ratio (RER) in $\mathrm{Oga}^{+/-}$ female mice, we also observed elevated RER in $\mathrm{Oga}^{+/-}$mice compared with $O g a^{+/+}$mice (ESM Fig. 6). These results indicate that $O g a^{+/-}$mice utilise more carbohydrates than do $\mathrm{Oga}^{+/+}$mice. Notably, Oga deficient mice generated by gene trap exhibited increased levels of $O$-GlcNAcylation and reduced OGT protein expression [11], which is consistent with the current hypothesis of $O$-GlcNAc homeostasis [35]. In contrast, both OGT protein expression and $O$-GlcNAcylation levels were elevated in OGA heterozygous mice produced by conditional disruption. We speculate that the different gene knock-out methods might lead to the different molecular and physiological changes in the two lines of $O g a$ heterozygous mice.

Ruan et al demonstrated that OGT in agouti-related peptide (AgRP) neurons regulate browning of WAT through modulation of $O$-GlcNAcylation of $\mathrm{Kv}$ channels [36]. They used AgRP neuron-specific Ogt-deleted mice. Decreased $O$ GlcNAcylation of Kv channels impairs neuronal firing in AgRP-Ogt knockout mice, which promotes browning of
WAT and protects against diet-induced obesity. In contrast, we suggest that $O g a$ heterozygosity intrinsically enhances brown adipocyte differentiation in $O g a^{+/-}$mice. However, we could not exclude the possibility that abnormally elevated $O$-GlcNAcylation in $O g a^{+/-}$AgRP neurons also disrupts neuronal firing, which contributes to browning of white fat in $\mathrm{Oga}^{+/-}$mice. This hypothesis is supported by many studies showing that a number of proteins involved in neuronal signalling and synaptic function are $O$-GlcNAcylated [37]. Consistent with this evidence, increased $O$-GlcNAcylation impairs synaptic function $[38,39]$. Further electrophysiological analysis using $\mathrm{Oga}^{+/-}$mice will be needed to test the hypothesis.

Understanding the mechanisms controlling thermogenesis is important for the prevention of weight gain and for understanding the development of obesity. Our results demonstrate that the reduced body weight of $\mathrm{Og}^{+/-}$mice results from increased energy expenditure through enhanced brown adipocyte differentiation in subcutaneous WAT. Based on our observations, changes in energy expenditure through the modulation of $O$-GlcNAc cycling may serve as a treatment for obesity and the metabolic syndrome.

Acknowledgements We thank J. H. Hur at UNIST-Olympus Biomedical imaging Center (UOBC), Ulsan, Republic of Korea, for technical support. We acknowledge the help with English language of professional editors from Textcheck (www.textcheck.com).

Duality of interest The authors declare that there is no duality of interest associated with this manuscript.

Funding This work was supported by a National Research Foundation of Korea (NRF) grant funded by the Korean Government (MSIP) (2014M3A9D8034456) and the Korean Government (MOE) (2013R1A1A2064434).

Author contribution statement YRY, HJJ and PGS contributed to the conception and design of the study. YRY, HJJ, YHL, GHL, SSC, ISK, YKS, JHC, DP, and AK contributed to the acquisition of data. YKS, HJJ, SSC, JHC, DP, AK, HL and SHR contributed to data analysis and interpretation. YRY drafted the manuscript, and all other authors contributed to the review and revision of intellectual content. All authors approved the final version to be published. PGS is the guarantor of this work.

\section{References}

1. Hart GW, Slawson C, Ramirez-Correa G, Lagerlof O (2011) Cross talk between O-GlcNAcylation and phosphorylation: roles in signaling, transcription, and chronic disease. Annu Rev Biochem $80: 825-858$

2. Yang X, Ongusaha PP, Miles PD et al (2008) Phosphoinositide signalling links O-GlcNAc transferase to insulin resistance. Nature 451:964-969

3. Whelan SA, Dias WB, Thiruneelakantapillai L, Lane MD, Hart GW (2010) Regulation of insulin receptor substrate 1 (IRS1)/AKT kinase-mediated insulin signaling by O-Linked beta- 
$\mathrm{N}$-acetylglucosamine in 3T3-L1 adipocytes. J Biol Chem 285:5204-5211

4. Dentin R, Hedrick S, Xie J, Yates J III, Montminy M (2008) Hepatic glucose sensing via the CREB coactivator CRTC2. Science 319:1402-1405

5. Housley MP, Rodgers JT, Udeshi ND et al (2008) O-GlcNAc regulates FoxO activation in response to glucose. J Biol Chem 283:16283-16292

6. Guinez C, Filhoulaud G, Rayah-Benhamed F et al (2011) OGlcNAcylation increases ChREBP protein content and transcriptional activity in the liver. Diabetes 60:1399-1413

7. Anthonisen EH, Berven L, Holm S, Nygard M, Nebb HI, Gronning-Wang LM (2010) Nuclear receptor liver X receptor is O-GlcNAc-modified in response to glucose. J Biol Chem 285:1607-1615

8. Lehman DM, Fu DJ, Freeman AB et al (2005) A single nucleotide polymorphism in MGEA5 encoding O-GlcNAc-selective N-acetylbeta-D glucosaminidase is associated with type 2 diabetes in Mexican Americans. Diabetes 54:1214-1221

9. Farook VS, Bogardus C, Prochazka M (2002) Analysis of MGEA5 on $10 \mathrm{q} 24.1-\mathrm{q} 24.3$ encoding the beta-O-linked Nacetylglucosaminidase as a candidate gene for type 2 diabetes mellitus in Pima Indians. Mol Genet Metab 77:189-193

10. Wu J, Bostrom P, Sparks LM et al (2012) Beige adipocytes are a distinct type of thermogenic fat cell in mouse and human. Cell 150:366-376

11. Yang YR, Song M, Lee $\mathrm{H}$ et al (2012) O-GlcNAcase is essential for embryonic development and maintenance of genomic stability. Aging Cell 11:439-448

12. Ji S, Park SY, Roth J, Kim HS, Cho JW (2012) O-GlcNAc modification of PPAR gamma reduces its transcriptional activity. Biochem Biophys Res Commun 417:1158-1163

13. Bullen JW, Balsbaugh JL, Chanda D et al (2014) Cross-talk between two essential nutrient-sensitive enzymes: O-GlcNAc transferase (OGT) and AMP-activated protein kinase (AMPK). J Biol Chem 289:10592-10606

14. Luo B, Parker GJ, Cooksey RC et al (2007) Chronic hexosamine flux stimulates fatty acid oxidation by activating AMP-activated protein kinase in adipocytes. J Biol Chem 282:7172-7180

15. Harms M, Seale P (2013) Brown and beige fat: development, function and therapeutic potential. Nat Med 19:1252-1263

16. Yuzwa SA, Macauley MS, Heinonen JE et al (2008) A potent mechanism-inspired O-GlcNAcase inhibitor that blocks phosphorylation of tau in vivo. Nat Chem Biol 4:483-490

17. Yang YR, Jang HJ, Yoon S et al (2014) OGA heterozygosity suppresses intestinal tumorigenesis in Apcmin/+ mice. Oncogenesis 3:e109

18. Cao W, Medvedev AV, Daniel KW, Collins S (2001) betaAdrenergic activation of p38 MAP kinase in adipocytes: cAMP induction of the uncoupling protein 1 (UCP1) gene requires p38 MAP kinase. J Biol Chem 276:27077-27082

19. Vosseller K, Wells L, Lane MD, Hart GW (2002) Elevated nucleocytoplasmic glycosylation by O-GlcNAc results in insulin resistance associated with defects in Akt activation in 3T3-L1 adipocytes. Proc Natl Acad Sci U S A 99:5313-5318

20. McClain DA, Lubas WA, Cooksey RC et al (2002) Altered glycandependent signaling induces insulin resistance and hyperleptinemia. Proc Natl Acad Sci U S A 99:10695-10699

21. Stubbs KA, Macauley MS, Vocadlo DJ (2009) A selective inhibitor Gal-PUGNAc of human lysosomal beta-hexosaminidases modulates levels of the ganglioside GM2 in neuroblastoma cells. Angew Chem 48:1300-1303
22. Macauley MS, Vocadlo DJ (2010) Increasing O-GlcNAc levels: an overview of small-molecule inhibitors of O-GlcNAcase. Biochim Biophys Acta 1800:107-121

23. Macauley MS, He YA, Gloster TM, Stubbs KA, Davies GJ, Vocadlo DJ (2010) Inhibition of O-GlcNAcase using a potent and cell-permeable inhibitor does not induce insulin resistance in 3T3L1 adipocytes. Chem Biol 17:937-948

24. Macauley MS, Bubb AK, Martinez-Fleites C, Davies GJ, Vocadlo DJ (2008) Elevation of global O-GlcNAc levels in 3T3-L1 adipocytes by selective inhibition of O-GlcNAcase does not induce insulin resistance. J Biol Chem 283:34687-34695

25. Macauley MS, Shan X, Yuzwa SA, Gloster TM, Vocadlo DJ (2010) Elevation of global O-GlcNAc in rodents using a selective OGlcNAcase inhibitor does not cause insulin resistance or perturb glucohomeostasis. Chem Biol 17:949-958

26. Xing D, Gong K, Feng W et al (2011) O-GlcNAc modification of NFkappaB p65 inhibits TNF-alpha-induced inflammatory mediator expression in rat aortic smooth muscle cells. PLoS One 6:e24021

27. Yang YR, Kim DH, Seo YK et al (2015) Elevated OGlcNAcylation promotes colonic inflammation and tumorigenesis by modulating NF-kappaB signaling. Oncotarget 20:12529-12542

28. Olivier-Van Stichelen S, Drougat L, Dehennaut V et al (2012) Serum-stimulated cell cycle entry promotes ncOGT synthesis required for cyclin D expression. Oncogenesis 1:e36

29. Hondares E, Rosell M, Diaz-Delfin J et al (2011) Peroxisome proliferator-activated receptor alpha (PPARalpha) induces PPARgamma coactivator 1alpha (PGC-1alpha) gene expression and contributes to thermogenic activation of brown fat: involvement of PRDM16. J Biol Chem 286:43112-43122

30. Ohno H, Shinoda K, Spiegelman BM, Kajimura S (2012) PPAR gamma agonists induce a white-to-brown fat conversion through stabilization of PRDM16 protein. Cell Metab 15:395-404

31. Kang S, Bajnok L, Longo KA et al (2005) Effects of Wnt signaling on brown adipocyte differentiation and metabolism mediated by PGC-1alpha. Mol Cell Biol 25:1272-1282

32. Ruan HB, Han X, Li MD et al (2012) O-GlcNAc transferase/host cell factor $\mathrm{C} 1$ complex regulates gluconeogenesis by modulating PGC-1alpha stability. Cell Metab 16:226-237

33. Ha JR, Hao L, Venkateswaran G, Huang YH, Garcia E, Persad S (2014) beta-Catenin is O-GlcNAc glycosylated at serine 23: implications for beta-catenin's subcellular localization and transactivator function. Exp Cell Res 321:153-166

34. Keembiyehetty C, Love DC, Harwood KR, Gavrilova O, Comly ME, Hanover JA (2015) Conditional knock-out reveals a requirement for O-linked N-Acetylglucosaminase (O-GlcNAcase) in metabolic homeostasis. J Biol Chem 290:7097-7113

35. Zhang Z, Tan EP, VandenHull NJ, Peterson KR, Slawson C (2014) O-GlcNAcase expression is sensitive to changes in O-GlcNAc homeostasis. Front Endocrinol 5:206

36. Ruan HB, Dietrich MO, Liu ZW et al (2014) O-GlcNAc transferase enables AgRP neurons to suppress browning of white fat. Cell 159:306-317

37. Khidekel N, Ficarro SB, Peters EC, Hsieh-Wilson LC (2004) Exploring the O-GlcNAc proteome: direct identification of OGlcNAc-modified proteins from the brain. Proc Natl Acad Sci U S A 101:13132-13137

38. Taylor EW, Wang K, Nelson AR et al (2014) O-GlcNAcylation of AMPA receptor GluA2 is associated with a novel form of long-term depression at hippocampal synapses. J Neurosci 34:10-21

39. Tallent MK, Varghis N, Skorobogatko Y et al (2009) In vivo modulation of O-GlcNAc levels regulates hippocampal synaptic plasticity through interplay with phosphorylation. J Biol Chem 284:174-181 